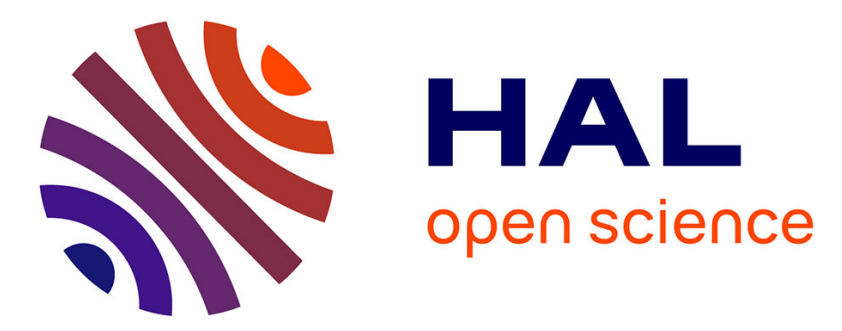

\title{
Definition of Essential Order on Descriptor Systems and its Bond Graph Determination
}

\author{
Julien Lagnier, Damien Eberard, Michaël Di Loreto, Didier Rémond, Wilfrid
}

Marquis-Favre

\section{- To cite this version:}

Julien Lagnier, Damien Eberard, Michaël Di Loreto, Didier Rémond, Wilfrid Marquis-Favre. Definition of Essential Order on Descriptor Systems and its Bond Graph Determination. ICBGM'16, International Conference on Bond Graph Modeling and Simulation, Jul 2016, Montréal, Canada. pp.133-142, 10.22360/SummerSim.2016.ICBGM.015 . hal-01863697

\section{HAL Id: hal-01863697 https://hal.science/hal-01863697}

Submitted on 13 Nov 2019

HAL is a multi-disciplinary open access archive for the deposit and dissemination of scientific research documents, whether they are published or not. The documents may come from teaching and research institutions in France or abroad, or from public or private research centers.
L'archive ouverte pluridisciplinaire HAL, est destinée au dépôt et à la diffusion de documents scientifiques de niveau recherche, publiés ou non, émanant des établissements d'enseignement et de recherche français ou étrangers, des laboratoires publics ou privés. 


\section{Definition of Essential Order on Descriptor Systems and its Bond Graph Determination}

\author{
Julien Lagnier \\ Engineer
}

Siemens PLM Software

La Cité International

84 quai Charles de Gaulle

Lyon, 69006, France

julien.lagnier@siemens.com

\author{
Damien Eberard \\ Associate Professor
}

Université de Lyon

Laboratoire Ampère (CNRS

UMR 5005), INSA-Lyon,

20 Avenue Albert Einstein

Villeurbanne, 69621, France

damien.eberard@insa-lyon.fr
Michael Di Loreto
Associate Professor

Université de Lyon

Laboratoire Ampère (CNRS UMR 5005), INSA-Lyon, 20 Avenue Albert Einstein Villeurbanne, 69621, France michael.di-loreto@insa-lyon.fr

\author{
Didier Rémond \\ Professor \\ Université de Lyon \\ LaMCoS (CNRS UMR 5259), \\ INSA-Lyon, \\ 20 Avenue des Sciences \\ Villeurbanne, 69621, France \\ didier.remond@insa-lyon.fr
}

\author{
Wilfrid Marquis-Favre \\ Professor \\ Université de Lyon \\ Laboratoire Ampère (CNRS UMR 5005), \\ INSA-Lyon \\ 20 Avenue Albert Einstein \\ Villeurbanne, 69621, France \\ wilfrid.marquis-favre@insa-lyon.fr
}

\begin{abstract}
In the context of model inversion, a structural invariant of the transfer matrix, the essential order, is used as a model specification criterion to determine the highest time-differentiation order reached by an output in the inverse model. Originally defined for linear timeinvariant (LTI) regular systems, we propose an algebraic extension to the class of LTI descriptor systems with regular matrix pencil and show that, from a descriptor system inversion viewpoint, this invariant characterizes the highest time-differentiation of the output like in the regular case. A bond graph determination procedure on the direct model is given using (bi)causal path inspection and the notion of families of different causal paths.
\end{abstract}

Key words Structural Analysis, Descriptor Systems, Structure at Infinity, Generalized Essential Order (GEO), Inverse Model, Bicausal Bond Graph.

\section{Introduction}

This paper is concerned with structural analysis of bond graph model in the context of model inversion, using a structural invariant of the transfer matrix called the essential order [1]. This invariant has originally been defined for LTI regular systems to solve the dynamic state feedback decoupling problem [2] and can be interpreted as the highest time-differentiation order reached by an output in the inverse model [2]. The determination of essential order uses the notion of structure at infinity of the transfer matrix, used in control theory among other for feedback decoupling or perturbation rejection [3], for which several graph-theoretical determination procedures have been developed for structured systems with directed graph (digraph) [4] [5] and bond graph [6] [7]. For model inversion problems of regular systems, the existing algebraic structural framework [8] has been derived in bond graph with the determination of structural inversibility conditions [9] [10], determination of relative degrees [6] [10] and determination of essential orders [11] [12] [13]. 
Almost all of these previous bond graph declinations use the notion of bicausality [14]. An application of a design methodology based on inverse model of regular system can be found in [15], including an highlight on the time-differentiation order of the output as a model specification criterion (Differentiability Criterion); this application, graphically declined on bond graph and motivation of the present works, gives structural bond graphic design model rules on the matching between model specification (given in term of differentiability of the outputs) and model implementation. The procedure provides also, graphically, structural information on the bond graph model for potential redesign in case of mismatch.

The present paper aims to generalize the existing bond graph framework on model inversion, especially the notion of essential order, to the class of LTI descriptor systems with regular matrix pencil. Such a system can be described by :

$$
\Sigma:\left\{\begin{aligned}
E \dot{x}(t) & =A x(t)+B u(t) \\
y(t) & =C x(t)+D u(t)
\end{aligned}\right.
$$

where $x(t) \in \mathbb{R}^{n}$ is the descriptor state vector, $u(t) \in \mathbb{R}^{m}$ the input vector, $y(t) \in \mathbb{R}^{p}$ the output vector and $E, A \in \mathbb{R}^{n \times n}, B \in \mathbb{R}^{n \times m}, C \in \mathbb{R}^{p \times n}, D \in \mathbb{R}^{p \times m}$, with a regular matrix pencil $(s E-A)$, that is $\operatorname{det}(s E-A) \neq 0$. Descriptor systems have been widely studied in the literature $[16,17,18]$ to answer to mechatronic modeling requirements to represent algebraic constraint equations that could occur in electrical networks [17] [18] or mechanical systems [19], leading to differential-algebraic equation (DAE) with singular matrix $E$ in (1). Descriptor systems are also usually called singular system, generalized state space systems or differential algebraic systems [18]. Structural analysis on linear descriptor system has been proposed graph-theoretically on digraph [20], on matroids [21] and on bond graph [22]. Importants bond graph contributions on structural analysis have also been proposed to study the class of hybrid systems that can also be brought into a descriptor form [23]. Descriptor system inversion is not yet completely formalized on bond graph, while it has been formalized within the algebraic framework in [24].

The present paper is organized as follows : the section 2 brings some background on structure at infinity of rational matrix and structure at infinity by row/column. Based on the structure at infinity of the (potentially non proper) transfer matrix, the section 3 proposes the definition of the Generalized Essential Order (GEO), a proof of its algebraic determination and its unicity, and its interpretation in the context of inverse model. Then a determination of the GEO is given on the direct (bi)causal bond graph model related to the descriptor system, using directed graph and the analogies between the two graphical approaches. An illustrative example is given in section 4 . The Conclusion suggests some directions of the future works.

\section{Preliminaries}

A rational fraction $g(s)$ is defined as the quotient of two polynomials, $g(s)=\frac{n(s)}{d(s)}$ with $n(s) \in \mathbb{R}[s], d(s) \in \mathbb{R}[s]$ and $d(s) \neq 0$. A rational fraction $g(s)=\frac{n(s)}{d(s)}$ is proper if $\operatorname{deg} d(s) \geq \operatorname{deg} n(s)$ and strictly proper if $\operatorname{deg} d(s)>$ $\operatorname{deg} n(s)$, where $\operatorname{deg}(\cdot)$ denotes the degree of $(\cdot)$. A rational (resp. proper rational, strictly proper rational) matrix has entries that are rational (resp. proper rational, strictly proper rational) fraction.

Definition 1. [3] Let $G(s)$ be a $(p \times m)$ rational (potentially not proper) matrix of rank $r$. Its Smith-McMillan factorization at infinity is defined as :

$$
G(s)=B_{1}(s)\left(\begin{array}{cc}
\Delta_{\infty}(s) & 0 \\
0 & 0
\end{array}\right) B_{2}(s)
$$

with $B_{1}(s)$ and $B_{2}(s)$ biproper matrices (i.e. proper and with proper inverse $), \Delta_{\infty}(s)=\operatorname{diag}\left(s^{-t_{1}}, \ldots, s^{-t_{r}}\right)$, with integers $t_{i} \in \mathbb{Z}$ such that $t_{1} \leq t_{2} \leq \ldots \leq t_{r}$. The sequence $\left\{t_{i}\right\}_{i=1, \ldots, r}$ is uniquely defined and is called the structure at infinity of $G(s)$ : when $t_{i} \geq 0$ (resp. $\left.t_{i}<0\right), t_{i}$ is the $i$-th order of a zero (resp. pole) at infinity.

The structure at infinity can also be determined with the examination of the minors of $G(s)$. Applied to the rational (potentially not proper) $p \times m$ full-row rank transfer function $T(s)$ of (1), given by $T(s)=C(s E-A)^{-1} B+D$, the structure at infinity of $T(s)$ can be formulated as follows.

Lemma 1. [21] The sum of the orders at infinity of $T(s)$ is given by:

$$
\sum_{j=1}^{i} t_{j}=-\delta_{i}=-\beta_{i}+d, \quad \text { for } i=1, \ldots, p
$$

With:

- $\delta_{i}$ is the highest degree of all $(i \times i)$ minors of $T(s)$.

- $d$ is the dimension of the dynamical part of the singular system, i.e. $d=\operatorname{deg} \operatorname{det}(s E-A)$. 
- $\beta_{i}$ is the highest degree of all $(n+i) \times(n+i)$ minors of the Rosenbrock matrix [16], also named system matrix, defined by $P(s)=\left(\begin{array}{cc}s E-A & B \\ -C & D\end{array}\right)$.

Additional definitions and properties on the behaviour at infinity of rational matrix are now introduced.

Definition 2. [2] Denote $g_{i}(s)$ the $i$-th column of $G(s)$. The order of the zero/pole at infinity of the $i$-th column is defined by the integer $k_{i} \in \mathbb{Z}$ as:

$$
k_{i}=\min _{k \in \mathbb{Z}}\left\{\lim _{s \rightarrow+\infty} s^{k} g_{i}(s)=g_{i} \neq 0\right\}, \quad \text { for } i=1, \ldots, m
$$

If $k_{i} \geq 0$ (resp. $\left.k_{i}<0\right), k_{i}$ is an order of a zero at infinity (resp. $-k_{i}$ is an order of pole at infinity) of the $i$-th column $g_{i}(s)$.

Definition 2 can be trivially generalized for the rows of $G(s)$.

Property 3. Let two matrices $M_{1}(s)$ and $M_{2}(s)$ linked by a left multiplication with a biproper matrix, $M_{1}(s)=$ $B(s) M_{2}(s)$ where $B(s)$ is a biproper rational matrix. The order of the zero/pole at infinity of the $i$-th column of $M_{1}(s)$ is equal to the order of the zero/pole at infinity of the $i$-th column of $M_{2}(s)$ and is uniquely defined.

We can use Property 3 to extend the definition of some known structural invariants on rational matrices, initially formulated on a rational proper case, and demonstrate their unicity.

Proposition 2. Let $T(s) \in \mathbb{R}^{p \times m}(s)$ be a full-row rank rational (not necessarily proper) matrix with $p \leq m$. Consider a factorization of $T(s)$ as:

$$
T(s)=\left[\begin{array}{ll}
R(s) & 0
\end{array}\right] B(s),
$$

where $R(s) \in \mathbb{R}^{p \times p}(s)$ is invertible and $B(s) \in \mathbb{R}^{m \times m}(s)$ is a rational biproper matrix. The orders of the zeros/poles at infinity of the columns of $R^{-1}(s)$ are uniquely defined.

Proof : The proof follows step by step the proof given in [2], applicable in the rational case.

\section{Generalized Essential Order (GEO)}

\subsection{Definition and algebraic determina- tion}

Using the definitions and demonstrations of the previous section, we now define the Generalized Essential Order (GEO) of (potentially non proper) rational matrices and we propose its algebraic determination.
Definition 4. Let $T(s) \in \mathbb{R}^{p \times m}(s)$ be a full-row rank rational (not necessarily proper) matrix, with $p \leq m$. We consider a factorization of $T(s)$ as $T(s)=[R(s) \quad 0] B(s)$ where $R(s) \in \mathbb{R}^{p \times p}(s)$ is invertible and $B(s) \in \mathbb{R}^{m \times m}(s)$ is a rational biproper matrix. The Generalized Essential Orders (GEO) of the rational matrix $T(s)$, denoted $\left\{n_{i e_{g}}\right\}_{i=1, \ldots, p}$, are defined as the orders of zeros/poles at infinity of the columns of $R^{-1}(s)$.

Using Proposition 2, it can be proved that GEO are uniquely defined.

The algebraic determination of GEO is based on ([2], Proposition 5) and generalizes it to the rational case (not necessarily proper). The procedure uses the structure at infinity of the rational matrix $T(s)$ to determine the structure at infinity of the column of $T^{-1}(s)$.

Proposition 3. Let $T(s) \in \mathbb{R}^{p \times m}(s)$ be a full-row rank rational matrix with $p \leq m$. The Generalized Essential Order (GEO), $\left\{n_{i e_{q}}\right\}_{i=1, \ldots, p}$, can be determined as follows:

$$
n_{i e_{g}}=\sum_{j=1}^{p} t_{j}-\sum_{j=1}^{p-1} \bar{t}_{i j}, \quad \text { for } i=1, \ldots, p
$$

where

- $p=\operatorname{rank} T(s)$, i.e. number of pole/zero at infinity of $T(s)$.

- $t_{j}$ is the order of the $j$-th pole/zero at infinity of $T(s)$.

- $\bar{t}_{i j}$ is the order of the $j$-th pole/zero at infinity of the rational matrix $T_{i}(s) \in \mathbb{R}^{(p-1) \times m}(s)$, obtained from $T(s)$ by removing its $i$-th row.

Proof $^{1}$ : The proof is done for $i=1$ and the procedure can be generalized by row permutations. We first consider a factorization of $T(s)$ as in (5), $T(s)=$ $\left[\begin{array}{ll}R(s) & 0\end{array}\right] B(s)$, where $R(s) \in \mathbb{R}^{p \times p}(s)$ is invertible and $B(s) \in \mathbb{R}^{m \times m}(s)$ is biproper. We write $R(s)=$ $\left[\frac{r_{1}(s)}{\bar{R}_{1}(s)}\right]$ where $r_{1}(s)$ is the first row of $R(s)$ and $\bar{R}_{1}(s) \in$ $\mathbb{R}^{(p-1) \times p}(s)$, of rank $(p-1)$. The Smith-McMillan factorization at infinity of $\bar{R}_{1}(s)$ can be written as:

$$
\bar{R}_{1}(s)=\bar{B}_{1}(s) \bar{\Delta}(s) \bar{B}_{2}(s)
$$

where $\bar{B}_{1}(s) \in \mathbb{R}^{(p-1) \times(p-1)}(s)$ and $\bar{B}_{2}(s) \in \mathbb{R}^{p \times p}(s)$ are biproper and $\bar{\Delta}(s)=\left[\begin{array}{ccc|c}s^{-\bar{t}_{11}} & & & 0 \\ & \ddots & & \vdots \\ & & s^{-\bar{t}_{1(p-1)}} & 0\end{array}\right]$.

${ }^{1}$ The proof follows the steps of ([2], Proposition 5) and check the validity of each step in the rational case (not necessarily proper). 
Then, $R(s)$ can be factorized as:

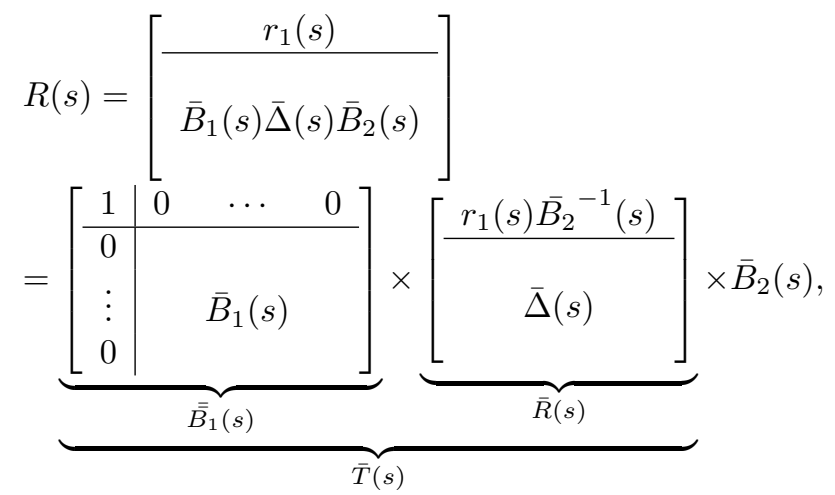

where $\overline{\bar{B}}_{1}(s) \in \mathbb{R}^{p \times p}(s)$ is biproper, and $\bar{R}(s), \bar{T}(s)$ are invertible. Using Property 3 , we see from $(8)$ that $R^{-1}(s)$ and $\bar{T}^{-1}(s)$ have the same orders of zero/pole at infinity by columns. The particular structure of biproper matrix $\overline{\bar{B}}_{1}(s)$ (and then $\left.\overline{\bar{B}}_{1}^{-1}(s)\right)$ implies that the first column of $\bar{T}^{-1}(s)$ equals the first column of $\bar{R}^{-1}(s)$, then have the same structure at infinity i.e. the same order of pole/zero at infinity by column. We also have (where $\operatorname{com}(\cdot)$ denotes the comatrix of $(\cdot))$ :

$$
\bar{R}^{-1}(s)=\frac{1}{\operatorname{det}(\bar{R}(s))} \operatorname{com}(\bar{R}(s))^{T}
$$

The first column of com $(\bar{R}(s))^{T}$ can then be written as $\left[\begin{array}{llll}0 & \cdots & 0 & (-1)^{(p+1)} \prod_{j=1}^{p-1} s^{-\bar{t}_{1 j}}\end{array}\right]^{T}$. In order to compute $\operatorname{det}(\bar{R}(s))$, we can rewrite $\bar{R}(s)$ from (8) as $\bar{R}(s)=\bar{B}_{1}^{-1}(s) R(s) \bar{B}_{2}^{-1}(s) ;$ additionally, the Smith McMillan factorization at infinity can be applied to the the full-rank square matrix $R(s)$ :

$$
R(s)=B_{3}(s) \Delta(s) B_{4}(s)
$$

where $B_{3}(s) \in \mathbb{R}^{p \times p}(s)$ and $B_{4}(s) \in \mathbb{R}^{p \times p}(s)$ are biproper matrices and $\Delta(s)=\operatorname{diag}\left(s^{-t_{1}}, \ldots, s^{-t_{p}}\right)$. Using (10), we have $\bar{R}(s)=\bar{B}_{1}^{-1}(s) B_{3}(s) \Delta(s) B_{4}(s) \bar{B}_{2}^{-1}(s)$ and then $\operatorname{det} \bar{R}(s)=b(s) \prod_{j=1}^{p} s^{-t_{j}}$, where $b(s)$ is a biproper function. We can eventually rewrite the first column of $\bar{R}^{-1}(s)$ as $\left[\begin{array}{llll}0 & \cdots & 0 & \frac{(-1)^{(p+1)} \prod_{j=1}^{p-1} s^{-\bar{t}_{1 j}}}{b(s) \prod_{j=1}^{p} s^{-t_{j}}}\end{array}\right]^{T}$. The order of the zero/pole at infinity of the first column of $\bar{R}^{-1}(s)$ is then equal to $\sum_{j=1}^{p-1}-\bar{t}_{i j}-\sum_{j=1}^{p}-t_{j}=$ $\sum_{j=1}^{p} t_{j}-\sum_{j=1}^{p-1} \bar{t}_{i j}$ and also stands for the order of the zero/pole at infinity of the first column of $\bar{T}^{-1}(s)$. By using (8) and reminding that $\bar{B}_{2}(s)$ is biproper, we can conclude that the structure at infinity of the columns of $R^{-1}(s)$ is equal to the one of $\bar{T}^{-1}(s)$. We can then deduce that the GEO $\left\{n_{i e_{g}}\right\}_{i=1, \ldots, p}$ of $T(s)$ can be determined as $n_{i e_{g}}=\sum_{j=1}^{p} t_{j}-\sum_{j=1}^{p-1} \bar{t}_{i j}, i=1, \ldots, p$, that ends the proof.

\subsection{Interpretation for LTI descriptor sys- tems and their inverses}

Using Definition 4 and Proposition 3, an interpretation of time-differentiation order that could occur in the inverse model of the descriptor system (1) can be given:

Lemma 4. We consider the LTI descriptor system (1), with its full-row rank rational transfer matrix $T(s) \in$ $\mathbb{R}^{p \times m}(s)$ with $p \leq m$. The Generalized Essential Order (GEO), $\left\{n_{i e_{g}}\right\}_{i=1, \ldots, p}$, of the $i$-th output of the system can be determined as $n_{i e_{g}}=\sum_{j=1}^{p} t_{j}-\sum_{j=1}^{p-1} \bar{t}_{i j}, i=1, \ldots, p$, where:

- $p=\operatorname{rank} T(s)$, i.e. number of pole/zero at infinity of the system $\Sigma$.

- $t_{j}$ is the order of the $j$-th pole/zero at infinity of the system $\Sigma$.

- $\bar{t}_{i j}$ is the order of the $j$-th pole/zero at infinity of the subsystem which has the $(p-1) \times m$ transfer function $T_{i}(s)$, obtained from $T(s)$ by removing its $i$-th row (i.e. by removing the $i$-th output).

In the case of LTI regular system (i.e. non-singular $E$ in (1)) and with $D=0$, leading to strictly proper rational transfer matrix, one can check that Proposition 3 is equivalent to the original definition in [2]. For the sake of simplicity, we consider in the following reasoning a square system (i.e. $T(s) \in \mathbb{R}^{p \times p}(s)$ ); two different cases can occur for the structure at infinity by column of the transfer matrix $T^{-1}(s)$ of the inverse system (see Definitions 2 and 1):

- If the structure at infinity of the i-th column of $T^{-1}(s)$ is a pole, the order of this pole corresponds to the highest time-differentiation order of the output $y_{i}$ occurring in the inverse model.

- If the structure at infinity of the i-th column of $T^{-1}(s)$ is a zero, the order of this zero corresponds to the lowest time-integration order of the output $y_{i}$ occurring in the inverse model.

\subsection{Digraph determination of the system matrix determinant}

The GEO determination uses the sum of the orders of zero/pole at infinity of the system (Proposition 3), that can be determined with the (highest) degrees of the minors of the system matrix (Lemma 1). We first propose 
a digraph extension of the graph-theoretical determination of the system matrix determinant to the class of LTI descriptor systems with $p=m$ (that, to the best of our knowledge, does not exist so far for this class of system, while noticing that almost all of the background elements are established in $[20])^{2}$.

Proposition 5. Let $\mathcal{G}(\Sigma)$ be the directed graph related to the descriptor system (1). The determinant of the system matrix can be interpreted graph-theoretically as :

$$
\operatorname{det}\left(\begin{array}{cc}
s E-A & B \\
-C & D
\end{array}\right)=\sum_{k=0}^{n} \rho_{k}^{\{p\}} s^{n-k}
$$

where (denoting E-edges the edges between vertices of matrix E):

- $\mathcal{G}\left(\Sigma^{\prime}\right)$ is the supplemented digraph obtained from $\mathcal{G}(\Sigma)$ by adding feedback edges with weight -1 from each output to each input.

- $\rho_{k}^{\{p\}}$ is determined on $\mathcal{G}\left(\Sigma^{\prime}\right)$ as the sum of the weights of the spanning families (of disjoint cycles) of dimension $n+p+p$ (i.e. containing the $n$ state vertices and exactly $p$ feedback edges) and containing exactly $(n-k)$ E-edges. Each family's weight must be multiplied by

- a sign factor $(-1)^{(n-k)-d}$, where $(n-k)$ is the number of E-edges and $d$ is the number of disjoint cycles.

- a sign factor $(-1)^{\sigma}$, where $\sigma$ is the number of permutations to sequence the outputs in the same order as the initial output vector when the cycles of the family are ordered in the initial order of the input vertices.

Proof : The proof is based on several graphtheoretical procedures on digraph $[4,20]$ :

- The computation of determinant of a square matrix of dimension $n+p$ necessarily implies spanning families of disjoint cycles of dimension $n+p[4]$.

- As in the regular case, input and output vertices are considered "separately" (i.e. $p$ input vertices and $p$ output vertices) with mandatory feedback edges to eventually consider $p+p$ inputs/outputs vertices while keeping the computation rule for the determinant of a $n+p$ matrix [4]. This explains why we consider $n+p+p$ spanning families of disjoint cycles here.

${ }^{2}$ The definitions of the several directed graph (digraph) notions used in this section can be found in [20].
- The only difference in the computation of the determinant of a system matrix between the regular and the descriptor case concerns the characteristic polynomial part. The graph-theoretical determination of $\operatorname{det}(s E-A)$ is proposed in [20]: on the $d i$ graph $\mathcal{G}(s E-A)$ corresponding to the matrix pencil $(s E-A)$, the characteristic polynomial can be determined as $\operatorname{det}(s E-A)=\sum_{k=0}^{n} \rho_{k} s^{n-k}$, where $\rho_{k}$ is the sum of the weights of the spanning families of disjoint cycles involving exactly $(n-k)$ E-edges. The remaining part of the computation of system matrix determinant is unchanged with respect to [4], introducing the input/output vertices and leading to the computation of the $\rho_{k}^{\{p\}}$ summand.

- The permutation factor $(-1)^{(n-k)-d}$ is unchanged with respect to the computation of the numerator polynomial of the transfer function of a descriptor system given in [20] and the permutation factor $(-1)^{\sigma}$ is unchanged with respect to the regular case of system matrix determinant given in [4]. This ends the proof.

It is important to note that Proposition 5 is applicable to any $(n+i) \times(n+i)$ minors of the system matrix by selecting the appropriate set of $i$ input / $i$ output vertices. The highest degree of the minor of order $n+i$ of system matrix is therefore the spanning family of $n+i+i$ vertices that contains the highest number of $E$-edges on the supplemented digraph.

\subsection{Bond graph determination of the sys- tem matrix determinant}

The determination of the system matrix determinant of a LTI descriptor system on its bond graph representation is based on Proposition 5 and the existing correspondence between disjoint cycles on a supplemented digraph (i.e. containing feedback edges) and input/output different causal paths on the corresponding bond graph [9], still valid for descriptor systems. This allows to directly conclude the following result (with $p=m$ ): ${ }^{3}$

Proposition 6. On the bond graph model representation of system (1), the determinant of the system matrix can be computed as :

$$
\operatorname{det}\left(\begin{array}{cc}
s E-A & B \\
-C & D
\end{array}\right)=\sum_{k=-n_{D}}^{n_{I}} \rho_{k}^{\{p\}} s^{n_{I}-k}
$$

where:

\footnotetext{
${ }^{3}$ The definitions of the several bond graph notions used in this section can be found in [9] and the state space form of system (1) from its bond graph representation is given in [22].
} 
- $n_{I}$ (resp. $n_{D}$ ) is the number of storage elements in integral causality (resp. in derivative causality).

- $p$ is the number of outputs (and inputs).

- $\rho_{k}^{\{p\}}$ is the sum of the static gains of the families of $p$ input/output different causal paths, of order $k$. Each family's static gain must be multiplied by

- a sign factor $(-1)^{d}$, where $d$ is the number of cycles contained in the family.

- a sign factor $(-1)^{\sigma}$, where $\sigma$ is the number of permutations to sequence the outputs in the same order as the initial output vector when the $p$ input/output different causal paths of the family are ordered in the initial order of the inputs.

Several remarks can be done :

- The Proposition 6 is consistent with the one formulated for the case of bicausal bond graph in [13] and demonstrated by introducing the Laplace directed graph, consistent with the digraph representation of linear descriptor systems (leading to the generalized form of descriptor systems $[17,10])$.

- The Proposition 6 is applicable to any $(n+i) \times(n+i)$ minors of the system matrix by selecting the appropriate set of $i$ inputs $/ i$ outputs. Thus, the highest degree of the $(n+i) \times(n+i)$ minor $\left(\beta_{i}\right.$ in $\left.(3)\right)$ is determined on the bond graph by the minimal order $\omega_{i}$ that a family of $i$ inputs/outputs different causal paths can have, then $\beta_{i}=n_{I}-\omega_{i}$.

\subsection{Bond graph determination of GEO}

The Proposition 6 allows to determine the structure at infinity of a descriptor system on its bond graph representation by using the lemma 1 ; together with proposition 3, the GEO can be determined on the bond graph:

Proposition 7. On the bond graph model representation of system (1), the Generalized Essential Orders (GEO) can be determined as follows:

$$
n_{i e_{g}}=\omega_{p}-\omega_{p-1}^{(i)}, \quad \text { for } i=1, \ldots, p
$$

where:

- $\omega_{p}$ is the minimal order that a family of $p$ inputs/outputs different causal paths can have on the bond graph.

- $\omega_{p-1}^{(i)}$ is the minimal order that a family of $p-1$ inputs/outputs different causal paths can have on the bond graph without considering the $i$-th output (i.e. without consideration of the $i$-th detector $\left(D_{e}\right)$ or the $i$-th $D_{e} D_{f}$ on bicausal bond graph).
Proof : Using Proposition 6, Lemma 1 and Proposition 3 leads to :

$$
\begin{aligned}
n_{i e_{g}} & =\sum_{j=1}^{p} t_{j}-\sum_{j=1}^{p-1} \bar{t}_{i j} \\
& =-\left(n_{I}-\omega_{p}\right)+d-\left(-\left(n_{I}-\omega_{p}^{(i)}\right)+d\right) \\
& =\omega_{p}-\omega_{p-1}^{(i)}
\end{aligned}
$$

In the initial system as well as the one without consideration of a given output, the number of storage in integral causality $n_{I}$ is unchanged, so as the characteristic polynomial (thus $d$ is unchanged). That completes the proof.

Proposition 7 can be seen as a "natural" extension of the works in [12], where a determination of the essential order has been proposed on the bond graph for LTI regular systems.

\section{Example}

We consider a direct model described by the LTI descriptor system (15) and its bicausal bond graph representation (Figure 1). For the sake of simplicity, we take a very usual case where the number of storage in integral causality is equal to the degree of the characteristic polynomial (i.e. $n_{I}=d$ ).

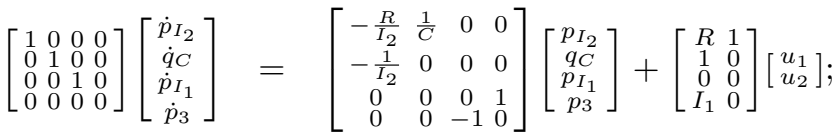

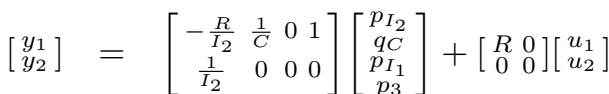

The system (15) is a descriptor system with regular pen-

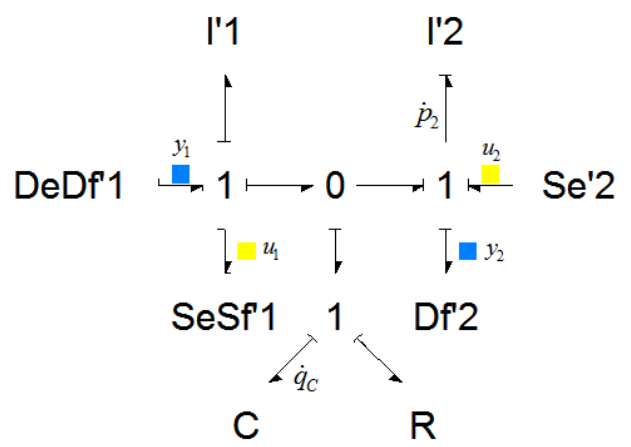

Figure 1: Bicausal bond graph representation of system (15) 
cil; its non-proper transfer function $T(s)$ is given in (16).

$$
\begin{aligned}
& T(s)= \\
& {\left[\begin{array}{cc}
\frac{s\left(C I_{1} I_{2} s^{2}+C I_{1} R s+C I_{2} R s+I_{1}+I_{2}\right)}{C I_{2} s^{2}+C R s+1} & -\frac{C R s+1}{C I_{2} s^{2}+C R s+1} \\
\frac{C R s+1}{C I_{2} s^{2}+C R s+1} & \frac{s C}{C I_{2} s^{2}+C R s+1}
\end{array}\right]}
\end{aligned}
$$

Using Proposition 7, the GEO determination on the bond graph 1 can be done with the following steps:

1. Determination of the structure at infinity of $T(s)$ from families of input/output different causal paths:

Determination of $t_{1}$ of $T(s)$ : The order of the first zero/pole at infinity of $T(s)$ can be determined as the highest degree of $1 \times 1$ minor of $T(s)$, or equivalently as the highest degree of $(n+1) \times(n+1)$ minor of $P(s)$. On the bond graph, according to Proposition 7 , we analyze the families of $p=1$ causal path Input $\rightarrow$ Output of order $k$. The bond graph 1 gathers 8 causal paths Input $\rightarrow$ Output (Figure 2). The minimal order of a family of $p=1$ input/output causal path is reached with the path $a_{o-1}$, with $k=-1$. In other words, $\min _{i, j}\left\{\omega\left(u_{i} \rightarrow y_{i}\right)\right\}=\omega_{a_{o-1}}=-1=\omega_{1}$. We thus find $t_{1}=\omega_{a_{o-1}}=-1$. We can also underline that the causal path $a_{o-1}$ is an input/output causal path from $u_{1}$ to $y_{1}$ and then corresponds to the fact that the transmittance (that represents here a $1 \times 1$ minor of $T(s))$ of highest degree of (16) is $t_{11}(s)$. We could also check that for the three other transmittances, the minimal orders of the input/output causal path are of order 1 , accordingly to (16) (i.e. $c_{o 1}$ for $t_{21}(s)$, $f_{o 1}$ for $t_{12}(s)$ and $e_{o 1}$ for $\left.t_{22}(s)\right)$.

Determination of $t_{2}$ of $T(s)$ : The order of the second zero/pole at infinity of $T(s)$ can be determined with the highest degree of $2 \times 2$ minor of $T(s)$, or equivalently with the highest degree of $(n+2) \times(n+2)$ minor of $P(s)$. On the bond graph, we look at families of $p=2$ different causal paths Input $\rightarrow$ Output of order $k$ (this family necessarily exists as the system is invertible, thus at least one family of $p=2$ input/output disjoint causal paths exists [10]). In this example, only one family of 2 Input $\rightarrow$ Output different causal path exists, formed by causal paths $a_{o-1}$ and $e_{o 1}$ (the uniqueness of a set of 2 inputs/outputs different causal path leads to the fact that these paths are disjoint, and insures in this case the inversibility of the system through these paths $[10,12])$. We can deduce that $\sum_{j=1}^{2} t_{j}=\omega_{a_{o-1}}+\omega_{e_{o 1}}=(-1)+(1)=0$ and then $t_{2}=1$.
2. Determination of the structure at infinity of submatrix $T_{i}(s)$ :

Structure at infinity of $T_{1}(s)$ : Without the consideration of the double detector $D_{e} D_{f}{ }^{\prime} 1$, we only analyze the set of causal path $u_{1} \rightarrow y_{2}$ and $u_{2} \rightarrow y_{2}$ (i.e. causal path $\left.c_{o 1}, e_{o 1}, g_{o 2}\right)$. The minimal order that (a set of one) causal path can have is 1 (path $c_{o 1}$ or path $e_{o 1}$ ). Thus, $\sum_{j=1}^{2-1} \bar{t}_{1 j}=1$.

Structure at infinity of $T_{2}(s)$ : Without considering the detector $D_{f}^{\prime} 2$, we only analyze the set of causal paths $u_{1} \rightarrow y_{1}$ and $u_{2} \rightarrow y_{1}$ (i.e. causal path $\left.a_{o-1}, b_{o 0}, d_{o 1}, f_{o 1}, h_{o 2}\right)$. The minimal order that (a set of one) causal path can have is -1 (path $a_{o-1}$ ). Therefore, we have $\sum_{j=1}^{2-1} \bar{t}_{2 j}=-1$.

3. Determination of Generalized Essential Orders (GEO) : Concerning output $y_{1}, n_{1 e_{g}}=\sum_{j=1}^{2} t_{j}-$ $\sum_{j=1}^{2-1} \bar{t}_{1 j}=(0)-(1)=-1$, and for output $y_{2}$, $n_{2 e_{g}}=\sum_{j=1}^{2} t_{j}-\sum_{j=1}^{2-1} \bar{t}_{2 j}=(0)-(-1)=+1$.

We can check the previous bond graphic steps with the algebraic determination of GEO on $T(s)$ by examination of the structure at infinity of $T(s)$ and its submatrices $T_{i}(s)$ (Proposition 3):

1. Determination of the structure at infinity of $T(s)$ from the degrees of its minors (Lemma 1):

Computation of $t_{1}$ of $T(s)$ from its $(1 \times 1) m i$ nors: from $(16)$, the highest degree of $(1 \times 1)$ minor of $T(s)$ is $\delta_{D} 1=+1$. Therefore $-\delta_{D 1}=t_{1}=-1$.

Computation of $t_{2}$ of $T(s)$ from its $(2 \times 2)$ minor: Noticing that $(2 \times 2)$ minor of $T(s)$ is $\operatorname{det} T(s)$, its (highest) degree is $\delta_{D_{2}}=0$. Then $-\delta_{D 2}=\sum_{j=1}^{2} t_{j}=0$ and $t_{2}=1$.

2. Determination of the structure at infinity of submatrices $T_{i}(s)$ :

Structure at infinity of $T_{1}(s): T_{1}(s)$ is obtained from $T(s)$ by removing its first row. Its structure at infinity is then its structure at infinity by row (Definition 2), i.e. a zero at infinity of order 1 . Then, we have $\sum_{j=1}^{2-1} \bar{t}_{1 j}=\bar{t}_{11}=1$.

Structure at infinity of $T_{2}(s): T_{2}(s)$ is obtained from $T(s)$ by removing its second row. Its structure at infinity is then a pole at infinity of order 1 . We then have $\sum_{j=1}^{2-1} \bar{t}_{2 j}=\bar{t}_{21}=-1$. 


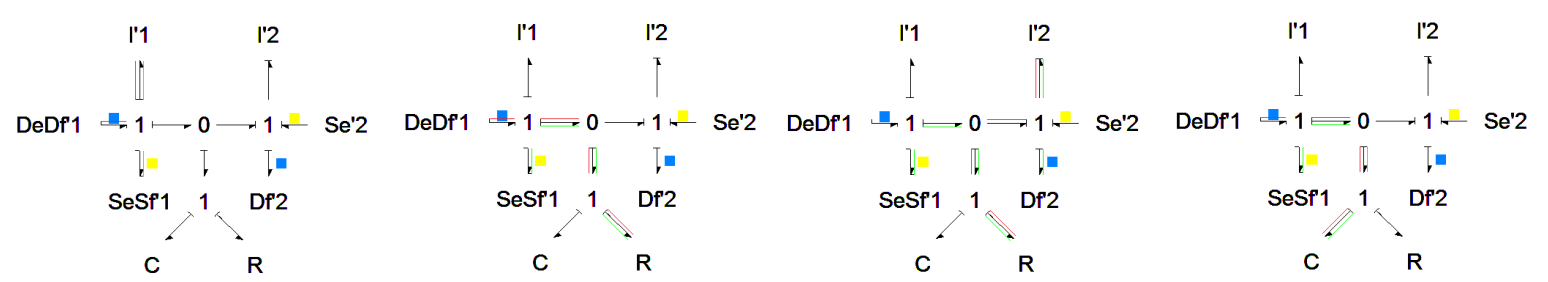

(a) Causal path

(b) Causal path

(c) Causal path

(d) Causal path

$u 1 \rightarrow y 1$ of order -1

$u 1 \rightarrow y 1$ of order 0

$\left(a_{o-1}\right)$

$\left(b_{o 0}\right)$

$u 1 \rightarrow$ y2 of order 1

$\left(c_{o 1}\right)$

$u 1 \rightarrow y 1$ of order 1

$\left(d_{o 1}\right)$

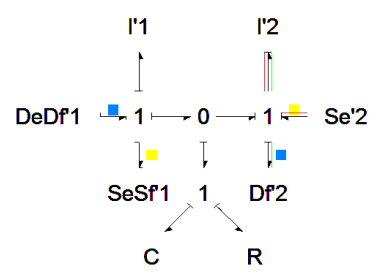

(e) Causal path

u2 $\rightarrow$ y2 of order 1

$\left(e_{o 1}\right)$

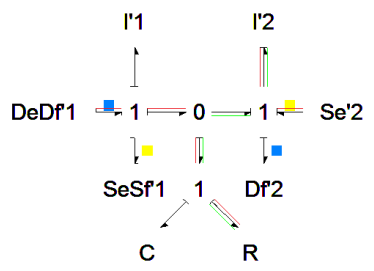

(f) Causal path

u2 $\rightarrow$ y1 of order 1

$\left(f_{o 1}\right)$

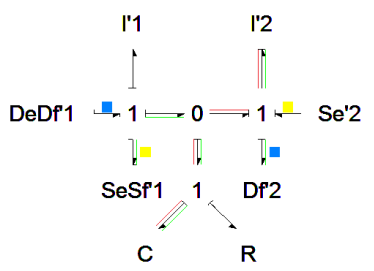

(g) Causal path

$u 1 \rightarrow$ y2 of order 2

$\left(g_{o 2}\right)$

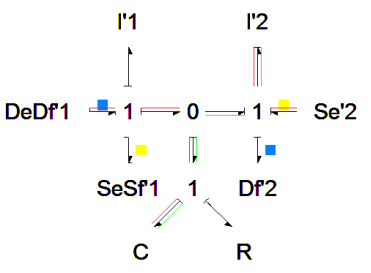

(h) Causal path

u2 $\rightarrow$ y1 of order 2

$\left(h_{o 2}\right)$

Figure 2: Causal paths Input $\rightarrow$ Output on bond graph 1

3. Determination of the GEO : Concerning output $y_{1}, n_{1 e_{g}}=\sum_{j=1}^{2} t_{j}-\sum_{j=1}^{2-1} \bar{t}_{1 j}=(0)-(1)=-1$, and for output $y_{2}, n_{2 e_{g}}=\sum_{j=1}^{2} t_{j}-\sum_{j=1}^{2-1} \bar{t}_{2 j}=$ $(0)-(-1)=+1$.

The algebraic determination of the structure at infinity of the system as well as the GEO are consistent with the bond graphic determination. We can also directly check the previous results with the algebraic definition on $T^{-1}(s)$ (i.e. Definition 4 applied on $T^{-1}(s)$ given in (17), symbolically computed from (16)), then requiring the development of the inverse model.

$$
\begin{aligned}
& T^{-1}(s)= \\
& {\left[\begin{array}{cc}
\frac{s C}{C I_{1} s^{2}+C R s+1} & \frac{C R s+1}{C I_{1} s^{2}+C R s+1} \\
-\frac{C R s+1}{C I_{1} s^{2}+C R s+1} & \frac{s\left(C I_{1} I_{2} s^{2}+C I_{1} R s+C I_{2} R s+I_{1}+I_{2}\right)}{C I_{1} s^{2}+C R s+1}
\end{array}\right]}
\end{aligned}
$$

The Definition 2 of the orders of zero/pole at infinity by column applied to $T^{-1}(s)$ leads to the following determination of the GEO (Definition 4) of $T(s)$ :

- $n_{1 e_{g}}=-1$ (i.e. the structure at infinity of the first column of $T^{-1}(s)$ is a zero at infinity of order 1$)$, and

- $n_{2 e_{g}}=1$ (i.e. the structure at infinity of the second column of $T^{-1}(s)$ is a pole at infinity of order 1$)$.
As a consequence for our application, the inverse model exhibits no time-differentiation of the first output, $y_{1}(t)$, in the expression of its inputs $u_{1}(t)$ and $u_{2}(t)\left(y_{1}(t)\right.$ will be integrated at least one time) and a maximal order of time-differentiation of 1 for the second output $y_{2}(t)$. Building the inverse model (figure 3$)^{4}$, the GEO could also be retrieved afterwards in the equation set (18).

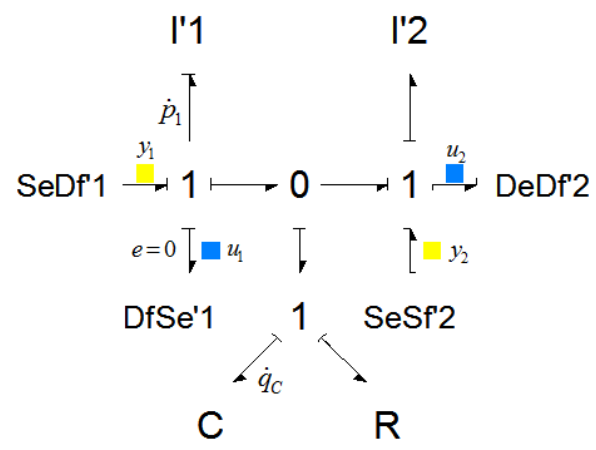

Figure 3: Bicausal bond graph representation of the inverse model of system (15)

\footnotetext{
${ }^{4}$ The direct model 1 contains bicausality assignments; thus the inverse model 3 explicitly details the source-sensor $[14,10]$ on the 1 junction related to storage element $I_{1}$, noticing that the effort is zero on the source-sensor $D_{f} S_{e}^{\prime} 1$ on figure 3 .
} 


$$
\begin{aligned}
\dot{p}_{1}(t) & =-\frac{R}{I_{1}} p_{1}(t)-\frac{1}{C} q_{C}(t)+y_{1}(t)+R y_{2}(t) \\
\dot{q}_{C}(t) & =\frac{1}{I_{1}} p_{1}(t)-y_{2}(t) \\
u_{1}(t) & =\frac{1}{I_{1}} p_{1}(t) \\
u_{2}(t) & =-\frac{R}{I_{1}} p_{1}(t)-\frac{1}{C} q_{C}(t)+R y_{2}(t)+I_{2} \dot{y}_{2}(t)
\end{aligned}
$$

\section{Conclusion}

We have proposed in this paper a generalization of the notion of essential order to the class of LTI descriptor system (1), introducing the Generalized Essential Orders (GEO) for non-proper rational matrices and proposing an algebraic determination based on their structure at infinity. In the context of descriptor system inversion, we have shown that this invariant characterizes the highest time-differentiation of the output like in the regular case. One of the interest of the approach lies in the fact that the determination is done on the transfer matrix of the system, even though the definition is on its inverse. Declined on bond graph, the procedure allows to give criteria on the time-differentiation order of the outputs of the model without building explicitly its inverse. In our opinion, the future interesting directions to our works could be a formalization of inversion of a bicausal bond graph, an extension of the digraph construction procedure from bicausal bond graph or structural determination of impulsive modes on bicausal bond graphs (directly linked to the determination of GEO).

\section{Acknowledgments}

This work has been done in the context of the MODRIO project. The authors would like to thanks the MODRIO team, Sébastien FURIC and Nicolas ORAND.

\section{References}

[1] C. Commault, J. Descusse, J. M. Dion, J. F. Lafay, and M. Malabre, "New decoupling invariants: the essential orders," International Journal of Control, vol. 44, no. 3, pp. 689-700, 1986.

[2] C. Commault and J. M. Dion, "Some structural invariants within the transfer matrix approach," in 1986 25th IEEE Conference on Decision and Control, pp. 1284-1288, Dec 1986.

[3] J. M. Dion and C. Commault, "Smith - memillan factorizations at infinity of rational matrix functions and their control interpretation," Systems $\&$ Control Letters, vol. 1, no. 5, pp. 312 - 320, 1982.

[4] K. J. Reinschke, Multivariable control : a graphtheoretic approach. Lecture notes in control and information sciences, Berlin, New York: SpringerVerlag, 1988. 236 pages.

[5] C. Commault, J. M. Dion, and A. Perez, "Disturbance rejection for structured systems," IEEE Transactions on Automatic Control, vol. 36, pp. 884-887, Jul 1991.

[6] A. Rahmani, C. Sueur, and G. Dauphin-Tanguy, "On the infinite structure of systems modelled by bond graph: feedback decoupling," in IEEE International Conference on Systems, Man, and Cybernetics, 1996., vol. 3, pp. 1617-1622 vol.3, Oct 1996.

[7] J. Bertrand, C. Sueur, and G. Dauphin-Tanguy, "On the finite and infinite structures of bond-graph models," in IEEE International Conference on Systems, Man, and Cybernetics, 1997. Computational Cybernetics and Simulation., vol. 3, pp. 2472-2477 vol.3, Oct 1997.

[8] L. M. Silverman, "Inversion of multivariable linear systems," IEEE Transactions on Automatic Control, vol. 14, pp. 270-276, Jun 1969.

[9] A. Rahmani, Etude structurelle des systèmes linéaires par l'approche Bond Graph. PhD thesis, Lille 1, 1993.

[10] R. Fotsu Ngwompo, Contribution au dimensionnement des systèmes sur des critères dynamiques et énergetiques: approche par bond graph. Thèse, Institut National des Sciences Appliquées (Lyon), 1997.

[11] M. El Feki, M. Di Loreto, E. Bideaux, D. Thomasset, and W. Marquis-Favre, "On the role of essential orders on feedback decoupling and model inversion : 
bond graph approach," in ECMS, (Nicosia, Cyprus), pp. 457-463, June 2008. Paper BG57.

[12] A. Jardin, Contribution à une méthodologie de dimensionnement des systèmes mécatroniques : analyse structurelle et couplage à l'optimisation $d y$ namique. Thèse, Institut National des Sciences Appliquées (Lyon, France), Jan. 2010.

[13] M. El Feki, Analyse et synthèse de tolérance pour la conception et le dimensionnement des systèmes mécatroniques. Thèse, Ecole Centrale de Lyon (France), July 2011.

[14] P. Gawthrop, "Bicausal bond graphs," in Proceedings of the International Conference On Bond Graph Modeling and Simulation (ICBGM'95), vol. 27 of Simulation Series, pp. 83-88, 1995.

[15] W. Marquis-Favre and A. Jardin, Bond Graph Modelling of Engineering Systems: Theory, Applications and Software Support, ch. Bond Graphs and Inverse Modeling for Mechatronic System Design, pp. 195226. New York, NY: Springer New York, 2011.

[16] H. H. Rosenbrock, "Structural properties of linear dynamical systems," International Journal of Control, vol. 20, no. 2, pp. 191-202, 1974.

[17] G. Verghese, B. Levy, and T. Kailath, "A generalized state-space for singular systems," IEEE Transactions on Automatic Control, vol. 26, pp. 811-831, Aug 1981.

[18] L. Dai, Singular Control Systems. Secaucus, NJ, USA: Springer-Verlag New York, Inc., 1989. 301 pages.

[19] K. Murota, Systems Analysis by Graphs and Matroids. Springer-Verlag Berlin Heidelberg, 1987. 284 pages.

[20] K. J. Reinschke, "Graph-theoretic approach to symbolic analysis of linear descriptor systems," Linear Algebra and its Applications, vol. 197 198, pp. 217 244, 1994.

[21] K. Murota and J. van der Woude, "Structure at infinity of structured descriptor systems and its applications," SIAM Journal on Control and Optimization, vol. 29, no. 4, pp. 878-894, 1991.

[22] A. Mouhri, A. Rahmani, and G. Dauphin-Tanguy, "Symbolic determination of generalized state equation for singular system modelled by bond graph," CSCC'99 Proceedings, pp. 2811-2816, 1999.
[23] J. Buisson and H. Cormerais, "Descriptor systems for the knowledge modelling and simulation of hybrid physical systems," in JESA Journal européen des systèmes automatisés, vol. 32, pp. 1047-1072, march 1998.

[24] F. L. Lewis, "Inversion of descriptor systems," in American Control Conference, 1983, pp. 1153-1158, June 1983. 